\title{
Linear Dichroism Spectra of Doped Calcium Gallogermanate Crystals
}

\author{
Y. SHOPA* AND N. FTOMYN \\ Physics Department, Ivan Franko National University of Lviv \\ Kyrylo and Mefodiy St. 8, 79005, Lviv, Ukraine
}

\begin{abstract}
Polarized absorption and linear dichroism spectra of chromium and manganese doped gallogermanate $\left(\mathrm{Ca}_{3} \mathrm{Ga}_{2} \mathrm{Ge}_{4} \mathrm{O}_{14}\right)$ crystals were measured and analyzed. Electronic transitions were identified and crystal field parameters have been determined. The value of linear dichroism obtained at the wavelength of $633 \mathrm{~nm}$ was compared with polarimetric measuring data for chromium doped $\mathrm{Ca}_{3} \mathrm{Ga}_{2} \mathrm{Ge}_{4} \mathrm{O}_{14}$ crystals.
\end{abstract}

PACS numbers: 78.20.Ci, 78.20.Fm

\section{Introduction}

This paper presents the investigation of the linear dichroism (LD) (linear dichroism is difference between the principal extinction coefficients of crystals) in chromium and manganese doped $\mathrm{Ca}_{3} \mathrm{Ga}_{2} \mathrm{Ge}_{4} \mathrm{O}_{14}(\mathrm{CGG})$ crystals. It was known that calcium gallogermanate are very attractive materials due to their various optical properties [1-3]. These crystals have the space group P321 and the crystal structure consists of tetrahedron layers, perpendicular to the $c$-axis, between which there are layers formed by cations in distorted Thomson cubes [4]. In addition, these systems are uniaxial and optically positive (the refractive indices of extraordinary $\left(n_{\mathrm{e}}\right)$ and ordinary $\left(n_{\mathrm{o}}\right)$ waves are related according to $n_{\mathrm{e}}>n_{\mathrm{o}}$ ) and possess a significant optical birefringence (OB), LD and circular dichroism (CD) [4].

A complete study about CD phenomena in CGG crystals in the range of electronic transitions of structure defects was introduced in [5]. At the same time the CD and absorption spectra in the range $240-850 \mathrm{~nm}$ of the chromium doped CGG crystals were in detail investigated by authors [5,6] on samples cut normally to optic axis, therefore the data about LD are unfortunately not present in these researches.

As it was shown, serious experimental difficulties usually exist if one measures simultaneously different optical anisotropy parameters: optical activity $(\mathrm{OA}), \mathrm{LD}, \mathrm{CD}$ in optically birefringent crystals [7, 8]. High-accuracy polarimetric methods represent the most precise and convenient techniques for solving the mentioned crystal optical problems. The temperature dependence of $\mathrm{LD}$ and $\mathrm{OB}$ changes have been already simultaneously measured in

* corresponding author; e-mail: shopa@physics.wups.lviv.ua
[9] for chromium doped gallogermanate crystals at the fixed wavelength $(633 \mathrm{~nm})$ using indirect methods of the high accuracy polarimetry. On the other hand, the direct spectral measuring of LD permits to verify the correctness of the polarimetric technique applied to CGG systems in [9] and, in the same time, the spectral methods are very informative and effective in analysis of the physical properties of crystals [10].

\section{Results and discussion}

The crystal plates CGG:Cr ${ }^{3+}$ and CGG:Mn ${ }^{3+}$ were cut parallel to optical axis and had the thickness of 0.72 and $1.53 \mathrm{~mm}$, respectively. The absorption spectra were measured in the spectral interval $400-800 \mathrm{~nm}$ for the both CGG: $\mathrm{Cr}^{3+}$ and CGG:Mn ${ }^{3+}$ crystals at the $T=293 \mathrm{~K}$. In the same time, the values of LD $(\Delta m)$ and LD parameter $(E)$ were calculated using the equations

$$
\Delta m=\frac{\lambda}{4 \pi d} \ln \left(\frac{I_{y}}{I_{z}} \frac{I_{0 z}}{I_{0 y}}\right), \quad E=\frac{2 \pi}{\lambda} d \Delta m,
$$

where $I_{0 y}, I_{0 z}$ - intensity of incident light polarized parallel to the $y$ and $z$ axes; $I_{y}, I_{z}$ - intensity of emergent light; $\lambda$ - wavelength of light; $d$ - thickness of specimen.

The polarized absorption spectra of CGG: $\mathrm{Cr}^{3+}$ (Fig. 1a) consist of comparatively wide asymmetric bands. These spectra have been analysed in the framework of crystal field theory [11] after deconvolution onto the elementary contours of Gaussian shape. As it is known, a term of ground state ${ }^{4} F$ of the free ion $\mathrm{Cr}^{3+}$ (an electronic configuration $3 d^{3}$ ) is split into ${ }^{4} A_{2},{ }^{4} T_{2}$, ${ }^{4} T_{1}$ levels in the octahedral crystal field [11]. From this point of view, the bands with maxima $15835 \mathrm{~cm}^{-1}$ and $22540 \mathrm{~cm}^{-1}$ which were observed in the polarized absorption spectra (Fig. 1a) can be attributed to the spin allowed ${ }^{4} A_{2} \rightarrow{ }^{4} T_{2},{ }^{4} A_{2} \rightarrow{ }^{4} T_{1}$ electronic transitions, respectively. In the same time, one can note that the information about the peak position of these bands can 
be used to calculation of the magnitude of crystal field strength $D q$, and Racah parameter $B$ [12]. Moreover, in this case it has been found that the $D q \approx 1584 \mathrm{~cm}^{-1}$ $(0.197 \mathrm{eV})$, and $B \approx 684 \mathrm{~cm}^{-1}(0.085 \mathrm{eV})$. Therefore, we have a good agreement with the results for ratio $D q / B$ obtained in [6]. Besides, using the analysis of the absorption and CD spectra obtained in [6], weakly intensive bands with the maxima $20540 \mathrm{~cm}^{-1}$ and $17965 \mathrm{~cm}^{-1}$ can be probably associated with $\mathrm{Cr}^{4+}$ and $\mathrm{Cr}^{3+}$ ions (it is known that chromium impurity could be introduced as $\mathrm{Cr}^{3+}$ and $\mathrm{Cr}^{4+}$ ions into different structural sites in the CGG crystals [6, 13]).
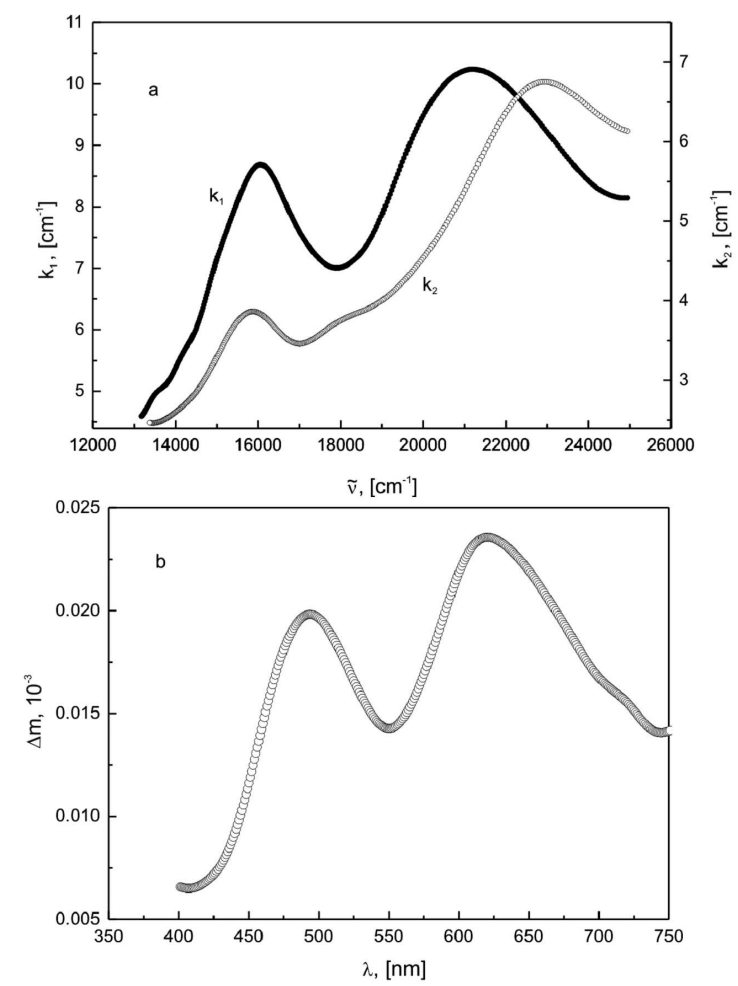

Fig. 1. Polarized absorption spectra (a) and linear dichroism spectrum (b) for CGG:Cr ${ }^{3+}$ crystals $\left(k_{1}, k_{2}\right.$ - absorption coefficients for two perpendicular polarizations of light).

Furthermore, there is a considerable shift of the maxima and the intensity difference between the most intensive bands $\left({ }^{4} A_{2} \rightarrow{ }^{4} T_{2},{ }^{4} A_{2} \rightarrow{ }^{4} T_{1}\right.$ transitions $)$ observed by means of the polarized absorption spectra (Fig. 1a). In other words, the linear dichroism occurs on the wide spectral interval (Fig. 1b) for chromium doped CGG crystals. In this case the reduction of local symmetry of the environment of $\mathrm{Cr}^{3+}$ ions in a crystal (the trivalent chromium ions occupy octahedral sites substituting $\mathrm{Ga}^{3+}$ ions in the positions with local symmetry 32 in the CGG: $\mathrm{Cr}^{3+}$ crystal $[6,13])$ can be a reason of $\mathrm{LD}$.

It is necessary to note that the linear dichroism parameter obtained in [9] remains unchangeable $(E=0.13$ and $\left.\Delta m=m_{z}-m_{y}=0.018 \times 10^{-3}\right)$ on temperature interval:
290-350 K. At the same time the value of LD obtained by means of the spectroscopic data of the present paper is approximately equal to $\Delta m=0.023 \times 10^{-3}$ for wavelength $633 \mathrm{~nm}$. As can be seen, these results indicate the correctness of indirect measuring method [9] and some deviation between both obtained $\Delta m$ values can be explained by higher precision of the polarimetric methods.

The similar spectral examination has been carried out for CGG: $\mathrm{Mn}^{3+}$ crystals. It is necessary to note that for $\mathrm{Mn}^{3+}$ ions in the octahedral crystal field (an electronic configuration $4 d^{4},{ }^{5} \mathrm{D}$ ) one spin allowed electronic transition ${ }^{5} E_{\mathrm{g}} \rightarrow{ }^{5} T_{2 \mathrm{~g}}$ [11] will be observed. Therefore, one wide band that was decomposed on the two components (using a procedure of derivative spectrophotometry [14]) with maxima: $21445 \mathrm{~cm}^{-1}, 18880 \mathrm{~cm}^{-1}$ has been observed in the polarized absorption spectra (Fig. 2a). These results coincide with data of the previous spectral investigation $[15,16]$. Likewise to chromium doped gallogermanate crystals, for CGG: $\mathrm{Mn}^{3+}$ the changes of bands intensity in the both polarized absorption spectra occur and, as a result, the LD is probably associated with non-octahedral symmetry of the environment of $\mathrm{Mn}^{3+}$ ions in the crystal.

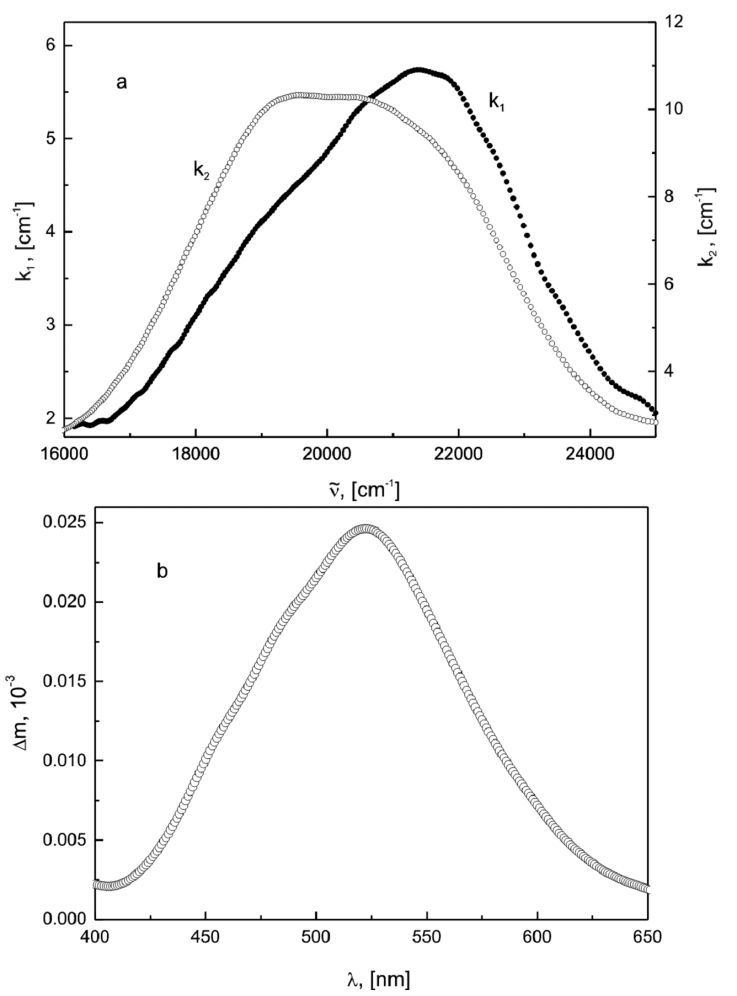

Fig. 2. Polarized absorption spectra (a) and linear dichroism spectrum (b) for CGG: $\mathrm{Mn}^{3+}$ crystal $\left(k_{1}, k_{2}\right.$ - absorption coefficients for two perpendicular polarizations of light).

Finally, the spectral dependence of LD for CGG:Mn ${ }^{3+}$ is represented in Fig. 2b. The value of $\Delta m$ for $\mathrm{He}-\mathrm{Ne}$ laser wavelength is defined to be equal to $0.003 \times 10^{-3}$, that is approximately 10 times less than the obtained 
for $\mathrm{Ca}_{3} \mathrm{Ga}_{2} \mathrm{Ge}_{4} \mathrm{O}_{14}: \mathrm{Cr}^{3+}$ and can be used in our next polarimetric investigations of these objects.

\section{Conclusions}

The polarized absorption and linear dichroism spectra for CGG: $\mathrm{Cr}^{3+}$ and CGG: $\mathrm{Mn}^{3+}$ are obtained and analyzed. It is shown that the appearance of linear dichroism in doped gallogermanate crystals is associated with distortion of octahedral symmetry of the environment of $\mathrm{Cr}^{3+}$ and $\mathrm{Mn}^{3+}$ ions. On the other hand, correlation between results obtained by means of the spectral (direct) and polarimetric (indirect) methods indicate the reasonableness of application of the principles of the high-accuracy polarimetry to investigation, the LD phenomena in the crystals. However, the polarimeter works usually on the fixed wavelength, therefore both methods can supplement each other.

\section{References}

[1] B. Mill, A. Klimenkova, B. Maximov, V. Molchanov, D. Pushcharovsky, Crystallogr. Rep. 52, 785 (2007).

[2] J. Wells, H. Gallagher, T. Han, J. Cryst. Growth 217, 302 (2000).

[3] H. Kong, J. Wang, H. Zhang, X. Yin, S. Zhang, Y. Liu, X. Cheng, L. Gao, X. Hu, M. Jiang, J. Cryst. Growth. 254, 360 (2003).

[4] A. Kaminskii, B. Mill, E. Sarkisov, Physics and Spectroscopy of Laser Crystals, Nauka, Moscow 1986 (in Russian).
[5] V. Burkov, E. Perederei, E. Fedotov, B. Mill, Yu. Pisarevski, Crystallogr. Rep. 53, 843 (2008).

[6] V. Burkov, A. Konstantinova, B. Mill, T. Veremeichik, Yu. Pyrkov, V. Orekhova, E. Fedotov, Crystallogr. Rep. 54, 613 (2009).

[7] J. Kobayashi, T. Asahi, M. Sakurai, M. Takahashi, K. Okubo, Y. Enomoto, Phys. Rev. B 53, 11784 (1996).

[8] J. Moxon, A. Renshaw, J. Phys., Condens. Matter 2, 6807 (1990)

[9] Y. Shopa, N. Ftomyn, Ukr. J. Phys. Opt. 7, 183 (2006).

[10] A.B.P. Lever, Inorganic Electronic Spectroscopy, Elsevier Science, Amsterdam 1984.

[11] D. Sviridov, R. Sviridova, Yu. Smirnov, Optical Spectra of Transition Metal Ions in Crystals, Nauka, Moscow 1976 (in Russian).

[12] M. Casalboni, A. Luci, U. Crassano, B. Mill, A. Kaminskii, Phys. Rev. B 49, 3781 (1994).

[13] K. Kaldybayev, A. Konstantinova, Z. Perekalina, Gyrotropy of Uniaxial Absorbing Crystals, ISPIN, Moscow 2000 (in Russian).

[14] G. Talsky, Derivative Spectrophotometry, WCH Verlagsgesellschaft, Weinheim 1994.

[15] B. Padlyak, O. Vlokh, B. Kuklinski, K. Sagoo, Ukr. J. Phys. Opt. 7, 1 (2006).

[16] A. Nosenko, R. Leshchuk, B. Padlyak, Zh. Prikl. Spektrosk. 59, 146 (1993). 